\title{
Reliability and validity of 4-metre gait speed in COPD
}

\author{
Samantha S.C. Kon ${ }^{1,3}$, Mehul S. Patel ${ }^{1,3}$, Jane L. Canavan ${ }^{1,3}$, Amy L. Clark², \\ Sarah E. Jones ${ }^{2}$, Claire M. Nolan ${ }^{1,2}$, Paul Cullinan', Michael I. Polkey ${ }^{1}$ and \\ William D-C. Man ${ }^{1,2}$
}

\begin{abstract}
Affiliations: ${ }^{1} \mathrm{NIHR}$ Respiratory Biomedical Research Unit, Royal Brompton and Harefield NHS Foundation Trust and Imperial College, London, ${ }^{2}$ Harefield Pulmonary Rehabilitation Unit, Royal Brompton and Harefield NHS Foundation Trust, Harefield, UK. ${ }^{3}$ These authors contributed equally.
\end{abstract}

Correspondence: S.S.C. Kon, Dept of Respiratory Medicine, Harefield Hospital, Hill End Road, Harefield, UB9 6JH, UK. E-mail: s.konarbht.nhs.uk

ABSTRACT In community-dwelling older adults, usual gait speed over $4 \mathrm{~m}$ (4MGS) consistently predicts greater risk of adverse health outcomes. The aims of the present study were to assess the reliability of the 4MGS and the relationship with established health outcome measures in chronic obstructive pulmonary disease (COPD).

Test-retest and interobserver reliability of the 4MGS were measured in 80 and 58 COPD patients, respectively. In $586 \mathrm{COPD}$ patients, the $4 \mathrm{MGS}$, as well as forced expiratory volume in $1 \mathrm{~s}(\mathrm{FEV} 1)$, the incremental shuttle walk (ISW), Medical Research Council (MRC) dyspnoea scale and St George's Respiratory Questionnaire (SGRQ) were measured. Participants were stratified according to "slow" $\left(<0.8 \mathrm{~m} \cdot \mathrm{s}^{-1}\right)$ or "normal" 4MGS $\left(\geqslant 0.8 \mathrm{~m} \cdot \mathrm{s}^{-1}\right)$.

Intra-class correlation coefficients for test-retest and interobserver reliability were 0.97 and 0.99 , respectively. There was a significant positive correlation between 4MGS with ISW $(\rho=0.78 ; \mathrm{p}<0.001)$ and a negative correlation with MRC dyspnoea scale and SGRQ ( $\rho=-0.55$ and $-0.44 ; \mathrm{p}<0.001$ for both). COPD patients with slow $4 \mathrm{MGS}$ had significantly reduced ISW and higher MRC dyspnoea scale and SGRQ than those with preserved walking speed, despite similar FEV1 \% predicted.

The 4MGS is reliable in COPD, correlates with exercise capacity, dyspnoea and health-related quality of life, and has potential as a simple assessment tool in COPD.

$@$ ERSpublications

The 4-metre gait speed test is reliable and valid as a simple assessment tool in chronic obstructive pulmonary disease http://ow.ly/kMHvX

Received: Oct 112012 | Accepted after revision: Nov 12 2012 | First published online: Dec 062012

Support statement: S.S.C. Kon and J.L. Canavan are supported by the Medical Research Council (UK). W.D-C. Man is supported by a National Institute for Health Research Clinician Scientist Award and a Medical Research Council (UK) New Investigator Research Grant (G1002113). This study was undertaken at the NIHR Respiratory Biomedical Research Unit at the Royal Brompton \& Harefield NHS Foundation Trust and Imperial College; M.I. Polkey's salary is part funded by this Biomedical Research Unit. The views expressed in this publication are those of the authors and not necessarily those of the NHS, The National Institute for Health Research nor the Department of Health.

Conflict of interest: Disclosures can be found alongside the online version of this article at www.erj.ersjournals.com 


\section{Introduction}

Usual gait speed measured over $4 \mathrm{~m}$ (4MGS) has been recommended as a potentially useful marker of multisystemic wellbeing in community-dwelling older adults [1]. A slow 4MGS is a consistent risk factor for disability, cognitive impairment, nursing home admission, falls and cardiovascular and all-cause mortality [1-4] in older adults who report no functional limitation. Moreover, it is reliable and quick to perform [5, 6]. 4MGS is also an established marker of exercise capacity in the elderly [7] and has been shown to correlate with other measures of function [8]. An expert working group recently identified the 4MGS as the most appropriate functional test for interventional trials in frail older adults [9]. A slow gait speed in the elderly is typically defined as a walking speed $<0.8 \mathrm{~m} \cdot \mathrm{s}^{-1}[1,4]$, which corresponds to a reduction in the median life expectancy for most ages [4].

Most studies of 4MGS have been in community-dwelling older adults, a proportion of whom are likely to have respiratory disease. However, the $4 \mathrm{MGS}$ has not been validated specifically in a chronic obstructive pulmonary disease (COPD) population. The use of a reliable, validated and easy to perform assessment tool to evaluate functional performance and predict adverse outcomes in COPD is attractive. Although field walking tests such as the 6-min walk test and incremental shuttle walk (ISW) test are well validated, they show a learning effect and require space, which limits widespread use in some clinical settings.

Therefore, the aims of this study were to assess the reliability of the 4MGS and its association with established outcome measures of exercise capacity, dyspnoea and health-related quality of life in COPD. A further aim was to describe the clinical characteristics of COPD patients with a slow 4MGS in comparison to those with preserved $4 \mathrm{MGS}$.

\section{Methods}

Subjects

Patients diagnosed with COPD according to Global Initiative for Chronic Obstructive Lung Disease (GOLD) guidelines [10] were recruited from outpatient respiratory clinics at Harefield Hospital (Harefield, UK). Exclusion criteria included unstable cardiac disease, exacerbation within the preceding 4 weeks, being unable to walk $4 \mathrm{~m}$ or a predominant neurological limitation to walking (e.g. significant hemiplegia). All participants gave informed consent and the study was approved by the West London and the LondonCamberwell St Giles Research Ethics Committees (11/H0707/2 and 11/LO/1780, respectively).

\section{Measurement of 4 MGS}

4MGS was measured by one of seven trained operators. All operators followed the gait speed component of the Short Physical Performance Battery (SPPB) protocol available from the National Institute on Aging (www.grc.nia.nih.gov/branches/ledb/sppb/). To perform the test, a flat unobstructed course was identified, either in a clinic assessment room or hospital corridor, and $4 \mathrm{~m}$ marked out with tape. A walk was demonstrated to each participant prior to starting the test, but participants themselves did not perform a practice walk. The participant was positioned with the toes just touching the start line. The following standardised instructions were given: "This is our walking course. I want you to walk to the other end of the course at your usual speed, just as if you were walking down the street to go to the shops. Walk all the way past the other end of the tape before you stop. Ready...begin". Timing with a stopwatch started when the participant began to move, not on the command "begin". Timing was stopped when the participant's first foot completely crossed the 4-m line. The walk test was repeated without rest and the faster of the two times was used to calculate the 4MGS, expressed in $\mathrm{m} \cdot \mathrm{s}^{-1}$. Participants were allowed to use their normal walking aids (e.g. cane). Oxygen users were provided with extra long tubing connected to a concentrator or immovable cylinder.

\section{Test-retest and interobserver reliability}

80 COPD patients underwent 4MGS measurement by the same operator on two occasions $24-48 \mathrm{~h}$ apart. 58 patients had 4MGS measured simultaneously by two observers on the same occasion.

\section{Cross-sectional study}

4MGS was measured in a convenience sample of 586 COPD patients. Further measurements included the ISW expressed in metres and \% predicted [11, 12], St George's Respiratory Questionnaire (SGRQ) [13], Medical Research Council (MRC) dyspnoea scale [14] and spirometry. These were performed by other investigators to ensure that the operators who measured 4MGS were blinded to these results. Comorbidities were recorded on the Charlson Index [15] and the recently validated COPD-specific comorbidity test index [16]. The participants were then stratified according to 4MGS, with "slow" walkers defined as a 4MGS $<0.80 \mathrm{~m} \cdot \mathrm{s}^{-1}$ and "preserved" defined as a $4 \mathrm{MGS}$ of $\geqslant 0.80 \mathrm{~m} \cdot \mathrm{s}^{-1}$, as recommended by expert consensus groups $[1,17]$. 


\section{Data analysis}

Data analyses and graphical presentations were performed using GraphPad Prism 5 (GraphPad Software, San Diego, CA, USA) or SPSS version 19 (IBM, Armonk, NY, USA). Single measure intra-class correlation coefficients (ICCs) of consistency and random effect were calculated with $95 \%$ confidence intervals to examine test-retest and interobserver reliability. Measurement variability was determined by calculating the standard error of measurement (SEM) and percentages of the standard errors of the measurements (SEM $\%$ ). Bland-Altman plots were plotted to demonstrate the $95 \%$ limits of agreement. Spearman's rank correlation was used to quantify the association between 4MGS and other measures. Between-group differences were assessed using unpaired t-tests or Mann-Whitney U-tests for nonparametric data. A p-value $<0.05$ was considered significant.

\section{Results}

Reliability

The 4MGS showed excellent test-retest and interobserver reliability, with ICC values of 0.97 (95\% CI 0.95 0.98 ) and 0.99 (95\% CI 0.98-0.99), respectively. Measurement variability was low, with SEM $1.4 \%$ for interobserver and $1.5 \%$ for test-retest reliability. Similarly, no systematic variability was demonstrated with narrow limits of agreement between occasions ( 0.135 to -0.125$)$ or observers ( 0.047 to -0.067$)$ (fig. 1$)$. Mean difference between test occasions was $0.005 \mathrm{~m} \cdot \mathrm{s}^{-1}$ and between observers was $-0.01 \mathrm{~m} \cdot \mathrm{s}^{-1}$.

\section{Cross-sectional measurement of 4MGS}

Baseline characteristics are shown in table 1. Sixty-seven, thirty-three and four patients used a walking aid only, ambulatory oxygen only or both, respectively. There was a strong positive correlation between 4MGS and ISW (table 2 and fig. 2a). Inspection of figure 2a shows a curvilinear relationship (second-order polynomial) between $4 \mathrm{MGS}$ and ISW (goodness of fit: $\mathrm{R}^{2}=0.59$ ) with the curve flatter at low ISW values, suggesting a floor effect to the ISW. 4MGS was also negatively correlated with age, MRC dyspnoea scale and SGRQ (activity and impact domains, total score in fig. 2b). There was a weak but statistically significant relationship with forced expiratory volume in $1 \mathrm{~s}(\mathrm{FEV} 1) \%$ pred $(\rho=0.10 ; \mathrm{p}=0.01)$ (fig. $2 \mathrm{c})$ and body mass index (BMI) $(\rho=-0.09 ; \mathrm{p}=0.03)$.

\section{Clinical characteristics related to slow gait speed}

193 patients had a slow 4MGS and 393 had preserved 4MGS. Participants with slow 4MGS were slightly older and had higher BMI, but there were no differences in sex distribution or FEV1 \% pred. However, large deficits in exercise capacity and health status were observed in the slow 4MGS group in ISW distance and SGRQ total score (table 3).

\section{Discussion}

We have demonstrated that usual 4MGS shows excellent test-retest and interobserver reliability in COPD. Furthermore, in a cohort of stable COPD patients, 4MGS was significantly correlated with measures of exercise capacity, health status and dyspnoea, supporting concurrent validity for the measure. Stratification according to "slow" 4MGS identified significant impairment in exercise capacity and poor health-related quality of life.
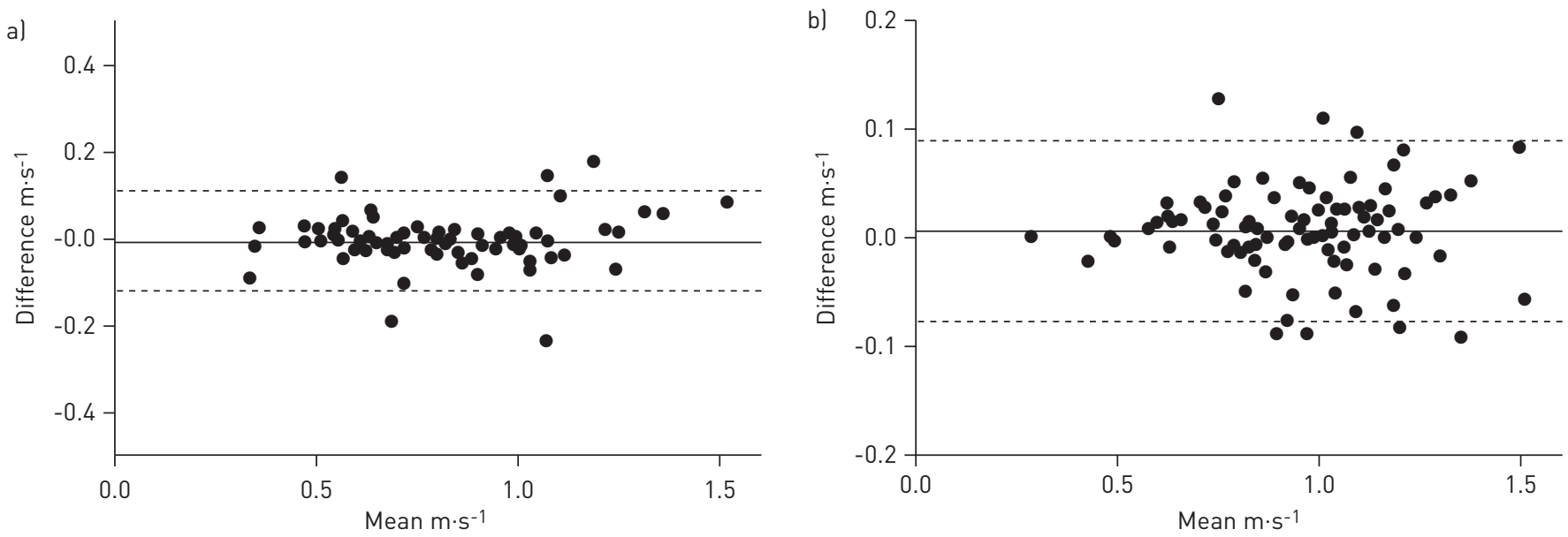

FIGURE 1 Bland-Altman plots for a) test-retest and b) interobserver reliability. 


\section{TABLE 1 Baseline characteristics of study subjects}

$\begin{array}{lc}\text { Subjects } & 586 \\ \text { Age years } & 70(63-76) \\ \text { Sex male/female } & 372 / 214 \\ \text { Height } \mathbf{~ m} & 1.67(1.58-1.73) \\ \text { Weight } \mathbf{~ g g} & 73.0(61.8-86.9) \\ \text { Body mass index } \mathbf{~ k g} \cdot \mathbf{m}^{-2} & 26.8(23.2-31.2) \\ \text { FEV1 \% predicted } & 46(29-61) \\ \text { MRC dyspnoea score } & 3(2-4) \\ \text { ISW test m } & 190(100-293) \\ \text { ISW test \% predicted } & 31(16-49) \\ \text { SGRQ } & \\ \text { Symptoms } & 68.6(52.4-81.2) \\ \quad \text { Activity } & 72.8(59.5-87.3) \\ \text { Impact } & 37.1(24.5-52.8) \\ \quad \text { Total } & 53.7(41.4-66.4) \\ \text { Charlson index } & 1(1-2) \\ \text { COTE index } & 0(0-1) \\ \text { Exacerbations in previous year } & 2(1-3) \\ \text { Hospital days in previous year } & 0(0-4.25) \\ \text { Gait speed } \mathbf{m} \cdot \mathbf{s}^{-1} & 0.89(0.74-1.05)\end{array}$

Data are presented as $\mathrm{n}$ or median (interquartile range). FEV1: forced expiratory volume in $1 \mathrm{~s}$; MRC: Medical Research Council; ISW: incremental shuttle walk; SGRQ: St George's Respiratory Questionnaire; COTE: chronic obstructive pulmonary disease-specific comorbidity test.

\section{Critique of the method}

Although gait speed has been reported over a variety of distances, most investigators currently believe that $4 \mathrm{~m}$ is optimal for a field test [18], balancing a distance short enough to be feasible in most clinical settings, but long enough to ensure measurement accuracy. Test-retest reliability for 4MGS is better than that reported over an 8-foot course [19] and as reliable as over $6 \mathrm{~m} \mathrm{[20].} \mathrm{A} \mathrm{4-m} \mathrm{course} \mathrm{has} \mathrm{also} \mathrm{been} \mathrm{shown} \mathrm{to}$ be feasible in $90 \%$ of households [21], allowing the possibility of using the 4MGS as a tool in communityor home-based studies. Consistent with this, in our study, we observed very high ICCs for both test-retest and interobserver reliability. Our results were perhaps towards the better end of those previously reported $[8,19,20]$, and this may reflect differences in patient populations. It is important to note that reliable 4MGS

TABLE 2 Spearman's rank correlation ( $\rho$ ) to compare the relationship between 4-m gait speed and other health outcome measurements

\begin{tabular}{lcc} 
& $\boldsymbol{\rho}$ & p-value \\
\hline Age & -0.21 & $<0.001$ \\
Height & 0.03 & 0.40 \\
Weight & 0.01 & 0.86 \\
BMI & -0.09 & 0.03 \\
FEV \% predicted & 0.10 & 0.01 \\
MRC dyspnoea score & -0.55 & $<0.001$ \\
ISW test & 0.78 & $<0.001$ \\
ISW test \% predicted & 0.72 & $<0.001$ \\
SGRQ & & \\
Symptoms & -0.15 & 0.007 \\
Activity & -0.51 & $<0.001$ \\
Impact & -0.38 & $<0.001$ \\
$\quad$ Total & -0.44 & $<0.001$ \\
Charlson index & -0.12 & 0.004 \\
COTE index & -0.09 & 0.03
\end{tabular}

BMI: body mass index; FEV1: forced expiratory volume in $1 \mathrm{~s}$; MRC: Medical Research Council; ISW: incremental shuttle walk; SGRQ: St George's Respiratory Questionnaire; COTE: chronic obstructive pulmonary disease-specific comorbidity test. 

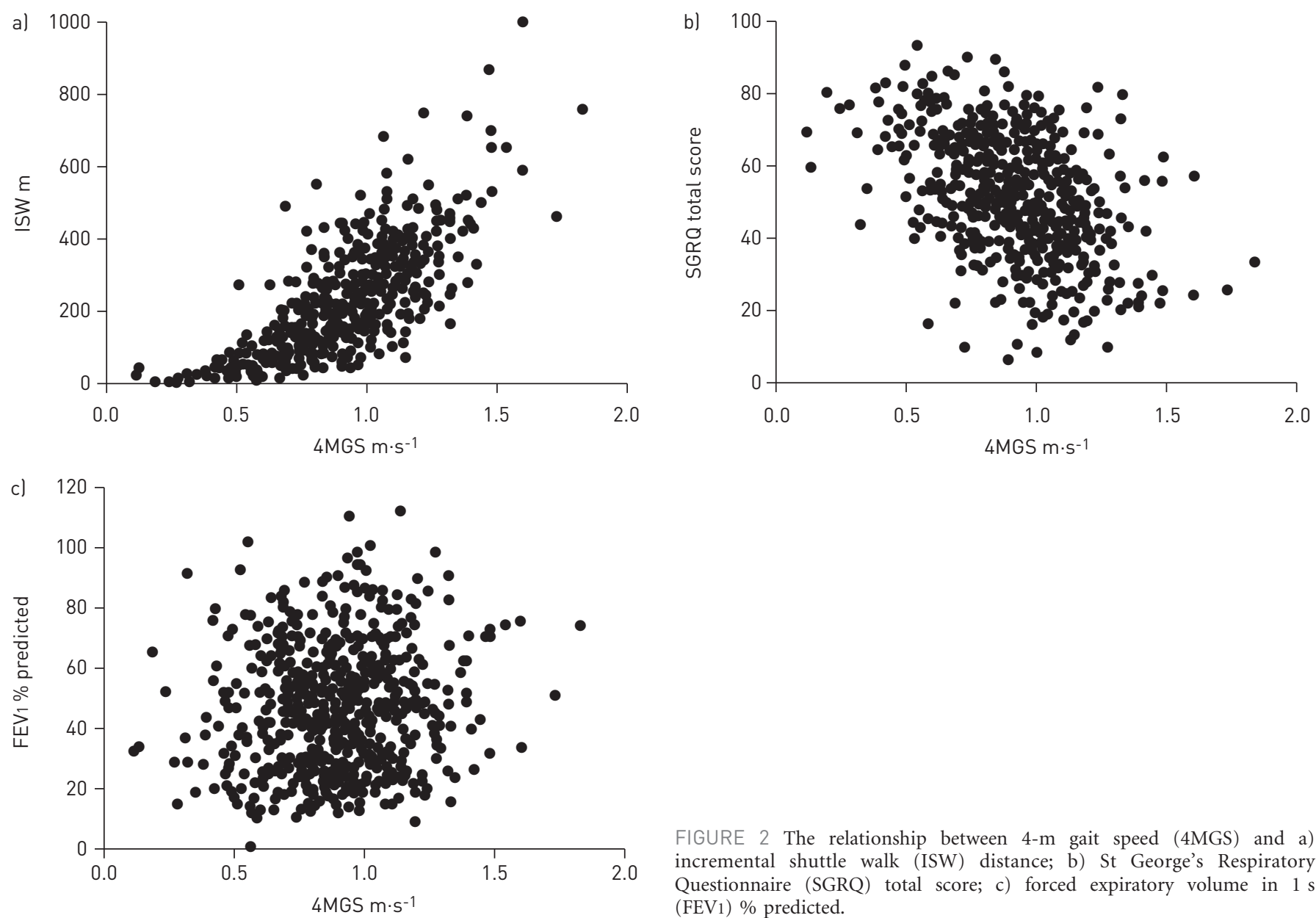

FIGURE 2 The relationship between 4 -m gait speed (4MGS) and a) incremental shuttle walk (ISW) distance; b) St George's Respiratory Questionnaire (SGRQ) total score; c) forced expiratory volume in $1 \mathrm{~s}$ (FEV1) \% predicted.

measurements can be made even by nonprofessional staff [6] and good reliability has been shown in frail patients or those with cognitive dysfunction $[19,20]$.

We asked patients to walk at their usual or comfortable speed in line with most previous reports. Although some investigators have modified the test by assessing maximal walking speed $[8,18]$, much of the data on the prognostic value of gait speed have focused on usual speed, and there is no evidence to indicate that measurement of maximal walking speed is any more reliable than usual speed [8,22]. However, we suspect that usual gait speed would be close to maximal speed in symptomatic severe COPD patients

Another consideration was the choice of gait speed cut-off. Several cut-off points for prediction of adverse health outcomes have been previously proposed. These have partly depended on the length of track, settings, the population being studied and the outcome of interest. For the 4MGS, "slow" is typically defined by expert consensus groups as a walking speed $<0.8 \mathrm{~m} \cdot \mathrm{s}^{-1}[1,17]$. Recently, in a pooled analysis of nine cohort studies of older adults (totalling 34485 individuals), years of remaining life for each sex and age increased as gait speed increased, with a gait speed of $0.8 \mathrm{~m} \cdot \mathrm{s}^{-1}$ at the median life expectancy for most ages [4]. Future longitudinal studies are required to validate the predictive value of this $4 \mathrm{MGS}$ cut-off in COPD populations, although, given the large differences in health outcomes demonstrated in our study, this cutoff appeared to be discriminatory.

\section{Significance of the findings}

The 4MGS as a single item tool has not been previously reported in COPD. RoIG et al. [23] recently reported self-selected gait speed in 21 COPD patients over a 5-m course, demonstrating a $20 \%$ reduction in speed compared with age-matched controls. ILGIN et al. [24] described significant associations between gait speed and lung function parameters and health-related quality of life in COPD, although gait speed was calculated from 6-min walk distance rather than from a short course. ANDERSSON et al. [25] showed high test-retest reliability when measuring walking speed in 49 COPD patients, although the 30-m course may limit generalisation of the findings. Evans et al. [26] demonstrated the validity of self-paced walking and 
TABLE 3 A comparison between chronic obstructive pulmonary disease (COPD) patients with low or normal 4-m gait speed (4MGS)

\begin{tabular}{lccc} 
& \multicolumn{2}{c}{ Gait speed } & p-value \\
\cline { 2 - 4 } & Slow & Normal & \\
\hline Subjects & 193 & 393 & $<0.001$ \\
Age years & $73(64-78)$ & $69(63-75)$ & 0.33 \\
Sex male/female & $100 / 93$ & $221 / 172$ & 0.72 \\
Height m & $1.65(1.58-1.74)$ & $1.58(1.41-1.67)$ & 0.46 \\
Weight kg & $74.6(61.0-89.0)$ & $72.1(62.0-86.0)$ & 0.01 \\
BMI kg.m ${ }^{-2}$ & $28.4(23.9-32.5)$ & $26.3(23.0-30.2)$ & 0.37 \\
FEV $\%$ predicted & $46(28-61)$ & $46(30-61)$ & $<0.001$ \\
MRC dyspnoea score & $4(4-5)$ & $3(2-4)$ & $<0.001$ \\
ISW distance $\mathbf{m}$ & $80(40-120)$ & $250(160-350)$ & $<0.001$ \\
ISW \% predicted & $14(7-24)$ & $41(28-57)$ & 0.11 \\
SGRQ & & & $<0.001$ \\
$\quad$ Symptoms & $71.5(54.3-82.2)$ & $67.8(51.6-80.2)$ & $<0.001$ \\
Activity & $85.8(72.8-92.5)$ & $66.3(53.5-79.8)$ & $<0.001$ \\
Impact & $47.9(32.1-58.4)$ & $34.0(22.3-47.1)$ & 0.02 \\
$\quad$ Total & $63.1(48.9-71.6)$ & $49.6(387-61.1)$ & 0.82 \\
Charlson index & $1(1-3)$ & $1(1-2)$ & 0.77 \\
COTE index & $0(0-1)$ & $0(0-1)$ & 0.004 \\
Exacerbations & $2(1-4)$ & $2(1-3)$ & $<0.001$ \\
Hospital days & $1(0-7)$ & $0(0-4)$ & \\
Gait speed m.s ${ }^{-1}$ & $0.67(0.56-0.74)$ & $0.99(0.89-1.12)$ & \\
\hline
\end{tabular}

Data are presented as $\mathrm{n}$ or median (interquartile range), unless otherwise stated. BMI: body mass index; FEV1: forced expiratory volume in $1 \mathrm{~s}$; MRC: Medical Research Council; ISW: incremental shuttle walk; SGRQ: St George's Respiratory Questionnaire; COTE: chronic obstructive pulmonary disease-specific comorbidity test.

responsiveness to pulmonary rehabilitation in $37 \mathrm{COPD}$ patients. Interestingly, although the test was performed in an enclosed, temperature-controlled $250-\mathrm{m}$ flat corridor, the repeatability was similar to that of $4 \mathrm{MGS}$ observed in our study. In addition to the considerably larger sample size, our data adds to the current literature by demonstrating that $4 \mathrm{MGS}$ correlates significantly to health outcome measures commonly used in COPD.

The 4MGS is also noted to be a single component of the Short Physical Performance Battery (SPPB). Previous data on the SPPB in COPD have been described in a single cohort [27-29], demonstrating lower (worse) SPPB scores in COPD patients compared with age-matched healthy controls, and an association between increased lean-to-fat ratio and preserved lung function with higher SPPB scores. This cohort was restricted to $40-65$ years of age and tended to have milder disease: $30 \%$ of patients were in the now-defunct GOLD stage $0[27,28]$. Our patients were older and more typical of COPD patients seen in hospital practice. Furthermore, we demonstrated that just one component of the SPPB had a strong association with clinically relevant outcomes. The 4MGS is quicker to perform, and previous studies have shown that the 4 MGS has similar associations and predictive abilities to the SPPB $[6,30]$.

The 4MGS has been frequently used in epidemiological studies of older adults due to its ease and speed of use. It has been shown to predict health status, functional decline, institutionalisation, onset of disability and mortality $[1,3,18,30]$. Other investigators have used gait speed as a functional outcome measure [31], and expert working groups (including representatives from the US Food and Drug Agency) have identified the $4 \mathrm{MGS}$ as the most appropriate functional test for interventional studies in frail older adults and sarcopenia $[9,32]$. The $4 \mathrm{MGS}$ has potential as an assessment tool in COPD. It is quick to perform, and cheap. Walking is familiar to the vast majority of adults, and the test is easy to understand; the 4MGS has been shown to be highly reliable even in people with cognitive dysfunction [20]. Furthermore, it requires little space, and the operator does not require any specialist training. These properties mean that the 4MGS could be adopted in almost any clinical environment (even at the bedside, or in primary care).

Our study demonstrated that 4MGS is reliable in COPD with excellent test-retest reliability. This is significant, particularly as current field tests of exercise capacity show a learning effect [11,33] and some, like the 6-min walk test, require a $30-\mathrm{m}$ course. Evidence of concurrent validity was demonstrated by showing significant associations with exercise capacity, health status and dyspnoea. By stratifying COPD 
patients according to 4MGS, we showed that the slow walking speed clinical phenotype exhibited significant deficits in exercise capacity, increased dyspnoea and poor health status in comparison to those with preserved walking speed. Interestingly, despite large differences in these outcome measures (which exceeded the commonly agreed minimum clinically important differences), there was no significant difference in FEV1 \% pred between the groups. Although 4MGS was shown to correlate negatively with age and BMI, it is unlikely that these were significant confounding factors for our results, given that there were still large differences in ISW \% pred (which corrects for age and BMI). Furthermore, 4MGS was not simply a reflection of comorbidity burden; we showed no or very weak relationships between 4MGS and validated comorbidity indices. We propose that 4MGS, like exercise capacity, reflects global wellbeing and captures the multisystemic effects of disease severity in COPD rather than pulmonary impairment alone.

\section{Limitations}

The study population was selective in that most were GOLD stage II-IV and symptomatic hospital outpatients. Our results need to be corroborated in other COPD populations (e.g. hospitalised inpatients or milder asymptomatic patients) in different settings. As the current study was cross-sectional, we could not evaluate the prognostic value of 4MGS in COPD. Longitudinal studies are required to assess the ability of 4MGS to predict clinically useful end-points. Although 4MGS was shown to correlate significantly with ISW, it is important to stress that the tool is not a measure of peak exercise capacity. However, it is an indicator of functional impairment, particularly in frail older patients [9], and has been previously used as a functional outcome measure in interventional pharmacological trials [31]. The curvilinear relationship between ISW and 4MGS (fig. 2a) suggests that the 4MGS may have a particular role in the assessment of poorly functioning individuals (i.e. there was a wide range in 4MGS in patients with very low ISW values). Further studies are needed to test the responsiveness of 4MGS to intervention in COPD. 4MGS may also have a role in rapidly screening COPD patients for extrapulmonary manifestations prior to more complex objective measurement of the outcome of interest. We did not make measurements of muscle mass or function, but this seems likely to be a fruitful area of research in COPD, particularly as expert consensus groups in sarcopenia and cachexia already recommend $4 \mathrm{MGS}$ as a screening tool $[17,32]$.

In summary, our study confirms that 4MGS is feasible and reliable in COPD, and correlates with measures of exercise capacity and health status. This demonstrates the potential utility of $4 \mathrm{MGS}$ as a simple assessment tool of multisystem wellbeing in COPD.

\section{Acknowledgements}

The authors would like to thank the Harefield Pulmonary Rehabilitation team (Harefield Hospital, Harefield, UK), for their help in collecting some of the data.

\section{References}

1 Abellan van Kan G, Rolland Y, Andrieu S, et al. Gait speed at usual pace as a predictor of adverse outcomes in community-dwelling older people. An International Academy on Nutrition and Aging (IANA) Task Force. J Nutr Health Aging 2009; 13: 881-889.

2 Dumurgier J, Elbaz A, Ducimetiere P, et al. Slow walking speed and cardiovascular death in well functioning older adults: prospective cohort study. BMJ 2009; 339: b4460.

3 Guralnik JM, Ferrucci L, Simonsick EM, et al. Lower-extremity function in persons over the age of 70 years as a predictor of subsequent disability. N Engl J Med 1995; 332: 556-561.

Studenski S, Perera S, Patel K, et al. Gait speed and survival in older adults. JAMA 2011; 305: 50-58.

5 Perera S, Mody SH, Woodman RC, et al. Meaningful change and responsiveness in common physical performance measures in older adults. J Am Geriatr Soc 2006; 54: 743-749.

6 Studenski S, Perera S, Wallace D, et al. Physical performance measures in the clinical setting. J Am Geriatr Soc 2003; 51: 314-322.

7 Buchner DM, Larson EB, Wagner EH, et al. Evidence for a non-linear relationship between leg strength and gait speed. Age Ageing 1996; 25: 386-391.

8 Steffen TM, Hacker TA, Mollinger L. Age- and gender-related test performance in community-dwelling elderly people: Six-Minute Walk Test, Berg Balance Scale, Timed Up \& Go Test, and gait speeds. Phys Ther 2002; 82: $128-137$.

9 Working Group on Functional Outcome Measures for Clinical Trials. Functional outcomes for clinical trials in frail older persons: time to be moving. J Gerontol A Biol Sci Med Sci 2008; 63: 160-164.

10 Rabe KF, Hurd S, Anzueto A, et al. Global strategy for the diagnosis, management, and prevention of chronic obstructive pulmonary disease: GOLD executive summary. Am J Respir Crit Care Med 2007; 176: 532-555.

11 Singh SJ, Morgan MD, Scott S, et al. Development of a shuttle walking test of disability in patients with chronic airways obstruction. Thorax 1992; 47: 1019-1024.

12 Probst VS, Hernandes NA, Teixeira DC, et al. Reference values for the incremental shuttle walking test. Respir Med 2012; 106: 243-248.

13 Jones PW, Quirk FH, Baveystock CM, et al. A self-complete measure of health status for chronic airflow limitation. The St George's Respiratory Questionnaire. Am Rev Respir Dis 1992; 145: 1321-1327.

14 Fletcher CM, Elmes PC, Fairbairn AS, et al. The significance of respiratory symptoms and the diagnosis of chronic bronchitis in a working population. $\mathrm{Br}$ Med J 1959; 2: 257-266. 
15 Charlson ME, Pompei P, Ales KL, et al. A new method of classifying prognostic comorbidity in longitudinal studies: development and validation. J Chronic Dis 1987; 40: 373-383.

16 Divo M, Cote C, de Torres JP, et al. Comorbidities and risk of mortality in patients with chronic obstructive pulmonary disease. Am J Respir Crit Care Med 2012; 186: 155-161.

17 Muscaritoli M, Anker SD, Argilés J, et al. Consensus definition of sarcopenia, cachexia and pre-cachexia: joint document elaborated by Special Interest Groups (SIG) "cachexia-anorexia in chronic wasting diseases" and "nutrition in geriatrics". Clin Nutr 2010; 29: 154-159.

18 Guralnik JM, Ferrucci L, Pieper CF, et al. Lower extremity function and subsequent disability: consistency across studies, predictive models, and value of gait speed alone compared with the short physical performance battery. J Gerontol A Biol Sci Med Sci 2000; 55: M221-M231.

19 Jette AM, Jette DU, Ng J, et al. Are performance-based measures sufficiently reliable for use in multicenter trials? Musculoskeletal Impairment (MSI) Study Group. J Gerontol A Biol Sci Med Sci 1999; 54: M3-M6.

20 Muñoz-Mendoza CL, Cabañero-Martínez MJ, Millán-Calenti JC, et al. Reliability of 4-m and 6-m walking speed tests in elderly people with cognitive impairment. Arch Gerontol Geriatr 2011; 52: e67-e70.

21 Simonsick EM, Maffeo CE, Rogers SK, et al. Methodology and feasibility of a home-based examination in disabled older women: the Women's Health and Aging Study. J Gerontol A Biol Sci Med Sci 1997; 52: M264-M274.

22 Bohannon RW. Comfortable and maximum walking speed of adults aged 20-79 years: reference values and determinants. Age Ageing 1997; 26: 15-19.

23 Roig M, Eng JJ, MacIntyre DL, et al. Deficits in muscle strength, mass, quality, and mobility in people with chronic obstructive pulmonary disease. J Cardiopulm Rehabil Prev 2011; 31: 120-124.

24 Ilgin D, Ozalevli S, Kilinc O, et al. Gait speed as a functional capacity indicator in patients with chronic obstructive pulmonary disease. Ann Thorac Med 2011; 6: 141-146.

25 Andersson M, Moberg L, Svantesson U, et al. Measuring walking speed in COPD: test-retest reliability of the 30metre walk test and comparison with the 6-minute walk test. Prim Care Respir J 2011; 20: 434-440.

26 Evans RA, Hill K, Dolmage TE, et al. Properties of self-paced walking in chronic respiratory disease; a patient-goal orientated assessment. Chest 2011; 140: 737-743.

27 Eisner MD, Blanc PD, Sidney S, et al. Body composition and functional limitation in COPD. Respir Res 2007; 8: 7.

28 Eisner MD, Blanc PD, Yelin EH, et al. COPD as a systemic disease: impact on physical functional limitations. Am J Med 2008; 121: 789-796.

29 Eisner MD, Iribarren C, Blanc PD, et al. Development of disability in chronic obstructive pulmonary disease: beyond lung function. Thorax 2011; 66: 108-114.

30 Montero-Odasso M, Schapira M, Soriano ER, et al. Gait velocity as a single predictor of adverse events in healthy seniors aged 75 years and older. J Gerontol A Biol Sci Med Sci 2005; 60: 1304-1309.

31 Goodman AD, Brown TR, Edwards KR, et al. A phase 3 trial of extended release oral dalfampridine in multiple sclerosis. Ann Neurol 2010; 68: 494-502.

32 Cruz-Jentoft AJ, Baeyens JP, Bauer JM, et al. Sarcopenia: European consensus on definition and diagnosis: Report of the European Working Group on Sarcopenia in Older People. Age Ageing 2010; 39: 412-423.

33 Sciurba F, Criner GJ, Lee SM, et al. Six-minute walk distance in chronic obstructive pulmonary disease: reproducibility and effect of walking course layout and length. Am J Respir Crit Care Med 2003; 167: 1522-1527. 